Maltepe Journal of Mathematics

ISSN:2667-7660, URL:HTTP://DERGIPARK.ORG.TR/TR/PUB/MJM

Volume III Issue 1 (2021), PAges 15-19. Doi:HtTPs://DOI.org/10.47087/MJM.896657

\title{
ON A MEAN METHOD OF SUMMABILITY
}

\author{
IBRAHIM ÇANAK* \\ *EGE UNIVERSITY, PHONE: +90 2323115418
}

Abstract. Let $p(x)$ be a nondecreasing real-valued continuous function on $R_{+}:=[0, \infty)$ such that $p(0)=0$ and $p(x) \rightarrow \infty$ as $x \rightarrow \infty$. Given a real or complex-valued integrable function $f$ in Lebesgue's sense on every bounded interval $(0, x)$ for $x>0$, in symbol $f \in L_{l o c}^{1}\left(R_{+}\right)$, we set

$$
s(x)=\int_{0}^{x} f(u) d u
$$

and

$$
\sigma_{p}(s(x))=\frac{1}{p(x)} \int_{0}^{x} s(u) d p(u), \quad x>0
$$

provided that $p(x)>0$.

A function $s(x)$ is said to be summable to $l$ by the weighted mean method determined by the function $p(x)$, in short, $(\bar{N}, p)$ summable to $l$, if

$$
\lim _{x \rightarrow \infty} \sigma_{p}(s(x))=l \text {. }
$$

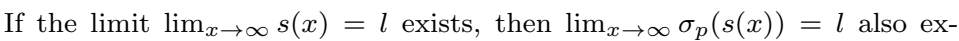
ists. However, the converse is not true in general. In this paper, we give an alternative proof a Tauberian theorem stating that convergence follows from summability by weighted mean method on $R_{+}:=[0, \infty)$ and a Tauberian condition of slowly decreasing type with respect to the weight function due to Karamata. These Tauberian conditions are one-sided or two-sided if $f(x)$ is a real or complex-valued function, respectively. Alternative proofs of some wellknown Tauberian theorems given for several important summability methods can be obtained by choosing some particular weight functions.

\section{INTRODUCTION}

Let $p(x)$ be a nondecreasing real-valued continuous function on $R_{+}:=[0, \infty)$. Throughout this paper, we assume that $p(0)=0$ and $p(x) \rightarrow \infty$ as $x \rightarrow \infty$. Given a real-valued integrable function $f$ in Lebesgue's sense on every bounded interval $(0, x)$ for $x>0$, in symbol $f \in L_{l o c}^{1}\left(R_{+}\right)$, we set

$$
s(x)=\int_{0}^{x} f(u) d u
$$

2020 Mathematics Subject Classification. Primary: 40E05; Secondaries: 40G05, 40A10.

Key words and phrases. summability by the weighted mean method; Tauberian conditions and theorems; slow decrease and oscillation with respect to a weight function.

(C)2019 Maltepe Journal of Mathematics.

Submitted on March 14th, 2021. Published on April 30th, 2021. Communicated by Huseyin CAKALLI.

The main results of this paper were presented at International Conference of Mathematical Sciences (ICMS 2020) to be held 17 June-21 June 2020 at Maltepe University, Istanbul, Turkey. 
and

$$
\sigma_{p}(s(x))=\frac{1}{p(x)} \int_{0}^{x} s(u) d p(u), \quad x>0
$$

provided that $p(x)>0$.

A function $s(x)$ is said to be summable to $l$ by the weighted mean method determined by the function $p(x)$, in short, $(\bar{N}, p)$ summable to $l$, if

$$
\lim _{x \rightarrow \infty} \sigma_{p}(s(x))=l .
$$

Clearly, if the ordinary limit

$$
\lim _{x \rightarrow \infty} s(x)=l
$$

exists, then 1.2 holds. However, the converse implication is not true in general. We may get the converse implication by adding some assumption(s) on $s(x)$, which is so-called Tauberian condition(s). Any theorem which states that convergence of 1.3 follows from 1.2 and a Tauberian condition is said to be a Tauberian theorem for summability by the weighted mean method.

A real-valued function $s(x)$ defined on $R_{+}$is said to be slowly decreasing with respect to $p$ if

$$
\lim _{\lambda \rightarrow 1^{+}} \liminf _{t \rightarrow \infty} \min _{t \leq x \leq T}(s(x)-s(t)) \geq 0,
$$

where

$$
T:=p^{-1}(\lambda p(t)), \quad t>0 .
$$

Note that the concept of slow decrease with respect to $p$ is due to Karamata [4].

It is easy to see that a real-valued function $s(x)$ is slowly decreasing with respect to $p$ if and only if for every $\epsilon>0$ there exist $t_{0}=t_{0}(\epsilon)>0$ and $\lambda=\lambda(\epsilon)>1$ such that $s(x)-s(t) \geq-\epsilon$ whenever $t_{0} \leq t \leq x \leq T$.

An equivalent reformulation of 1.4 can be given as follows (see Fekete and Moricz [1]):

$$
\lim _{\lambda \rightarrow 1^{-}} \liminf _{t \rightarrow \infty} \min _{T \leq x \leq t}(s(t)-s(x)) \geq 0,
$$

where $T$ is defined in 1.5 . It is easy to see that a real valued function $s(x)$ is slowly decreasing with respect to $p$ if and only if for every $\epsilon>0$ there exist $t_{1}=t_{1}(\epsilon)>0$ and $\lambda=\lambda(\epsilon)$ with $0<\lambda<1$ such that $s(t)-s(x) \geq-\epsilon$ whenever $t_{1} \leq T \leq x \leq t$.

A real-valued function $s(x)$ defined on $R_{+}$is said to be slowly decreasing if $(1.4)$ holds, where $p(x)=x$ for all $x>0$. Recall that the term "slow decrease" is introduced by Schmidt 7 for sequences of real numbers.

In [3, we obtained an alternative proof of Theorem 2.1 below when a Tauberian condition is of slowly decreasing type.

In this paper, we give an alternative proof a Tauberian Theorem stating that convergence follows from summability by weighted mean method over $R_{+}$and a Tauberian condition of slowly decreasing type with respect to the weight function, due to Karamata [4.

Alternative proofs of some well-known Tauberian theorems given for several important summability methods can be obtained by choosing some particular weight functions. 


\section{MAIN RESULTS}

By using proving techniques in [6], we give an alternative proof of the following Tauberian theorem [5] for the weighted mean summability of integrals of real-valued functions over $R_{+}$.

Theorem 2.1. Let $p(x)$ be a nondecreasing real-valued continuous function on $R_{+}$ such that $p(0)=0$ and $p(x) \rightarrow \infty$ as $x \rightarrow \infty$. If a real-valued function $f \in L_{l o c}^{1}\left(R_{+}\right)$ is such that (1.2) holds and its integral function $s(x)$ is slowly decreasing with respect to $p$, then (1.3) holds.

Proof. By the regularity of the summability method by the weighted mean, without loss of generalization, we assume that $l=0$. Assume that $s(x)$ does not converge to 0 as $x \rightarrow \infty$.

Then, we have either $\lim \sup _{x \rightarrow \infty} s(x)>0$ or $\liminf _{x \rightarrow \infty} s(x)<0$.

First, we assume that $\lim \sup _{x \rightarrow \infty} s(x)>0$. Then, there exist $\alpha>0$ and a sequence $\left(n_{i}\right)$ such that $s\left(n_{i}\right) \geq \alpha$ for all nonnegative integers $i$. Choosing $\epsilon=\frac{\alpha}{2}$ in the equivalent form of 1.4 , we find $\lambda>1$ and $t_{0} \geq 0$ such that $s(x) \geq s\left(n_{i}\right)-\frac{\alpha}{2} \geq \frac{\alpha}{2}$ for $t_{0} \leq n_{i}<x \leq m_{i}=p^{-1}\left(\lambda p\left(n_{i}\right)\right)$.

Since

$$
\begin{aligned}
\sigma_{p}\left(s\left(m_{i}\right)\right)-\frac{p\left(n_{i}\right)}{p\left(m_{i}\right)} \sigma_{p}\left(s\left(n_{i}\right)\right) & =\sigma_{p}\left(s\left(m_{i}\right)\right)-\frac{1}{\lambda} \sigma_{p}\left(s\left(n_{i}\right)\right) \\
& =\frac{1}{p\left(m_{i}\right)} \int_{n_{i}}^{m_{i}} s(u) d p(u)
\end{aligned}
$$

we have

$$
\begin{aligned}
\sigma_{p}\left(s\left(m_{i}\right)\right)-\frac{p\left(n_{i}\right)}{p\left(m_{i}\right)} \sigma_{p}\left(s\left(n_{i}\right)\right) & \geq \frac{\alpha}{2 p\left(m_{i}\right)} \int_{n_{i}}^{m_{i}} d p(u) \\
& =\frac{\alpha}{2}\left(1-\frac{1}{\lambda}\right)
\end{aligned}
$$

for $t_{0} \leq n_{i}<x \leq m_{i}=p^{-1}\left(\lambda p\left(n_{i}\right)\right)$. We conclude by 2.1 that $0 \geq \frac{\alpha}{2}\left(1-\frac{1}{\lambda}\right)$. This contradicts our assumption that $\lim _{\sup _{x \rightarrow \infty}} s(x)>0$. Then, we have

$$
\limsup _{x \rightarrow \infty} s(x) \leq 0 \text {. }
$$

Next, we assume that $\liminf _{x \rightarrow \infty} s(x)<0$. Then, there exist $\beta<0$ and a sequence $\left(n_{i}\right)$ such that $s\left(n_{i}\right) \leq \beta<0$ for all nonnegative integers $i$. Choosing $\epsilon=-\frac{\beta}{2}$ in the equivalent form of 1.4, we find $0<\lambda<1$ and $t_{1}=t_{1}(\epsilon)$ such that $s(x) \leq s\left(n_{i}\right)-\frac{\beta}{2} \leq \frac{\beta}{2}$ for $t_{1} \leq m_{i}=p^{-1}\left(\lambda p\left(n_{i}\right)\right)<x \leq n_{i}$.

Since

$$
\begin{aligned}
\sigma_{p}\left(s\left(n_{i}\right)\right)-\frac{p\left(m_{i}\right)}{p\left(n_{i}\right)} \sigma_{p}\left(s\left(m_{i}\right)\right) & =\sigma_{p}\left(s\left(n_{i}\right)\right)-\lambda \sigma_{p}\left(s\left(m_{i}\right)\right) \\
& =\frac{1}{p\left(n_{i}\right)} \int_{m_{i}}^{n_{i}} s(u) d p(u),
\end{aligned}
$$

we have

$$
\begin{aligned}
\sigma_{p}\left(s\left(n_{i}\right)\right)-\frac{p\left(m_{i}\right)}{p\left(n_{i}\right)} \sigma_{p}\left(s\left(m_{i}\right)\right) & \leq \frac{\beta}{2 p\left(n_{i}\right)} \int_{m_{i}}^{n_{i}} d p(u) \\
& =\frac{\beta}{2}(1-\lambda)
\end{aligned}
$$


for $t_{1} \leq m_{i}=p^{-1}\left(\lambda p\left(n_{i}\right)\right) \leq x \leq n_{i}$. We conclude by 2.3 that $0 \leq \frac{\beta}{2}(1-\lambda)$. This contradicts our assumption that $\liminf _{x \rightarrow \infty} s(x)<0$. Then, we have

$$
\liminf _{x \rightarrow \infty} s(x) \geq 0 .
$$

Combining 2.2 and (2.4) gives convergence of $s(x)$ to 0 as $x \rightarrow \infty$.

A real-valued function $s(x)$ defined on $\mathbf{R}_{+}$is said to be slowly increasing with respect to $p$ if $-s$ is slowly decreasing with respect to $p$.

Remark. Theorem 2.1 remains true if slow decrease of $s(x)$ with respect to $p$ is replaced by slow increase of $s(x)$ with respect to $p$.

For a complex-valued integrable function $f$ in Lebesgue's sense on every bounded interval $(0, x)$ for $0<x<\infty$, we have the following Tauberian theorem.

Theorem 2.2. Let $p(x)$ be a nondecreasing real-valued continuous function on $R_{+}$ such that $p(0)=0$ and $p(x) \rightarrow \infty$ as $x \rightarrow \infty$. If a complex-valued function $f \in$ $L_{\text {loc }}^{1}\left(R_{+}\right)$is such that (1.2) holds and its integral function $s(x)$ is slowly oscillating with respect to $p$, then (1.3) holds.

The proving technique in Theorem 2.1 is also valid for the proof of Theorem 2.2 .

We remind the reader that a complex-valued function $s(x)$ defined on $R_{+}$is said to be slowly oscillating with respect to $p$ (4]) if

$$
\lim _{\lambda \rightarrow 1^{+}} \limsup _{t \rightarrow \infty} \max _{t \leq x \leq T}|s(x)-s(t)|=0,
$$

where $T$ is defined as 1.5 .

It is easy to see that a real-valued function $s(x)$ is slowly oscillating with respect to $p$ if and only if for every $\epsilon>0$ there exist $t_{0}=t_{0}(\epsilon)>0$ and $\lambda=\lambda(\epsilon)>1 \mathrm{such}$ that $|s(x)-s(t)| \leq \epsilon$ whenever $t_{0} \leq t \leq x \leq T$.

An equivalent reformulation of 2.5 can be given as follows (see Fekete and Moricz [1]):

$$
\lim _{\lambda \rightarrow 1^{-}} \limsup _{t \rightarrow \infty} \max _{T \leq x \leq t}|s(t)-s(x)|=0,
$$

where $T$ is defined in 1.5 . It is easy to see that a real valued function $s(x)$ is slowly decreasing with respect to $p$ if and only if for every $\epsilon>0$ there exist $t_{1}=t_{1}(\epsilon)>0$ and $\lambda=\lambda(\epsilon)$ with $0<\lambda<1$ such that $|s(t)-s(x)| \leq \epsilon$ whenever $t_{1} \leq T \leq x \leq t$.

A complex-valued function $s(x)$ defined on $R_{+}$is said to be slowly oscillating if 2.5. holds, where $p(x)=x$ for all $x>0$.

Recall that the concept of slow oscillation was introduced by Hardy 2 for sequences of real numbers.

\section{PARTICULAR WEIGHTS}

Some particular choices of weight functions can lead to alternative proofs of some well-known Tauberian theorems given for several important summability methods. If $p(x)=x$ for all $x>0$, then weighted mean method $(\bar{N}, p)$ reduces to the Cesàro summability method. If $p(x)=\ln x$ for all $x \in[1, \infty)$ and zero for all $x \in[0,1)$, then then weighted mean method $(\bar{N}, p)$ reduces the harmonic mean method of first order. For other particular choices of the weight function $p$, we obtain the harmonic mean method of higher order. Our main Theorem 2.1 applies to all of these methods. 


\section{REFERENCES}

[1] Á. Fekete, and F. Móricz, Necessary and sufficient Tauberian conditions in the case of weighted mean summable integrals over $R_{+}$. II, Publ. Math. Debrecen. 67 (1-2) (2005) 65-78.

[2] G. H. Hardy, Theorems relating to the summability and convergence of slowly oscillating series, Proc. London Math. Soc.(2). 8 (1910) 310-320.

[3] Ç. Kambak, and İ. Çanak, An alternative proof of a Tauberian theorem for the weighted mean summability of integrals over $R_{+}$, Creat. Math. Inform. 29 (1) (2020) 45-50.

[4] J. Karamata, Sur les théorèms inverses de procédés de sommabilité, Hermann et Cie, Paris, 1937.

[5] F. Móricz, Necessary and sufficient Tauberian conditions in the case of weighted mean summable integrals over $R_{+}$, Math. Inequal. Appl. 7 (1) (2004) 87-93.

[6] A. Peyerimhoff, Lectures on summability, Springer, Berlin, 1969.

[7] R. Schmidt, Über divergente Folgen und lineare Mittelbildungen, Math. Z. 22 (1925) 89-152.

İBRAHIM ÇANAK,

Ege University, Department of Mathematics, Izmir, Turkey, Phone: +90 2323115418 , ORCID NumBer: 0000-0002-1754-1685

Email address: ibrahim.canak@ege.edu.tr 